P431 EFFECT OF TARGETED INTERVENTION ON CONDOM USE AND AMONG MEN WHO HAVE SEX WITH MEN (MSM) TAKING PREP IN NAIROBI KENYA

Maureen Akolo*., Unitid, Nairobi, Kenya

10.1136/sextrans-2019-sti.517

Background In 2017 Kenya became the second country in Africa to roll out PrEP. It prioritized Key population to access free PrEP services. Sex Workers Outreach Program (SWOP) a HIV/STI research center offers preventive, care and treatment services to female sex workers and MSM within Nairobi. In 2018 SWOP realized high STI cases among MSM on PrEP and introduced an intervention seeking to improve their STI prevention knowledge.

Methods An experimental study was carried out among HIV negative MSM enrolling into PrEP within SWOP clinics. Participants were randomized to control and intervention. Control group received PrEP services as prescribed by the government; the intervention group received targeted health education in addition to government prescribed services. Both arms were followed up for six months and screened for STIs at every visit. Data were collected at baseline and endline using structured questionnaire.

Results 168 MSM enrolled into study with 84(50\%) on each arm. At baseline condom use and STI Knowledge was low across both arms. At endline knowledge level had improved significantly in intervention arm compared to control arm with significance association to the targeted health education: Used K.Y Jelly to lubricate condom $\chi 2=4.983, \quad \mathrm{df}=1$, $\mathrm{P}=0.026$. Knew how to use male condoms Fisher's $<0.001$. Used condom consistently $\chi 2=0.92, \mathrm{df}=1, \mathrm{P}=0.337$. Negotiate for condom use $\chi 2=28.886, \mathrm{df}=1 \mathrm{P}<0.001$. Negotiated condom use with regular clients $\chi 2=8.839, \mathrm{df}=1, \mathrm{P}=0.003$. Refused sex when client refused condom use $\chi 2=11.483$, $\mathrm{df}=1, \mathrm{P}=0.001$. Syndromically STI cases at month one were $28 / 84(33.3 \%)$ in control, while 26/84 (30.9\%) in intervention arm. At month six cases were $16 / 38(42 \%)$ control and $7 / 59$ $(12 \%)$ in intervention arm.

Conclusion Targeted health education on condom use among MSM on PrEP is vital in averting STI cases.

Disclosure No significant relationships.

\section{P432 HIGH PREVALENCE AND INCIDENCE OF CURABLE STIS AMONG YOUNG WOMEN INITIATING PREP IN A TOWNSHIP IN SOUTH AFRICA}

${ }^{1}$ Katherine Gill ${ }^{*}{ }^{2}$ Connie Celum, ${ }^{2}$ Gabrielle Breen, ${ }^{2}$ Katherine Thomas, ${ }^{2}$ Jennifer Morton ${ }^{3}$ Jared Baeten, ${ }^{1}$ Eve Mendel, ${ }^{1}$ Menna Duyver, ${ }^{4}$ Linda-Gail Bekker. ${ }^{1}$ Desmond Tutu HIV Foundation, Desmond Tutu HIV Centre, Cape Town, South Africa; '2University of Washington, Seattle, USA; 3University of Washington, Medicine, Global Health, Epidemiology, Seattle, USA; ${ }^{4}$ University of Cape Town, Infectious Diseases, Cape Town, South Africa

\subsection{6/sextrans-2019-sti.518}

Background Sexually-transmitted infections (STIs) in adolescents and young women (AGYW) increase the risk of infertility and the risk of HIV acquisition. Recent data on STIs in African AGYW are limited because of syndromic management and lack of STI testing. PrEP programs that test for STIs can provide critical epidemiologic data to guide policies.

Methods Sexually-active HIV-uninfected AGYW (16-25 years) in a township near Cape Town, South Africa were enrolled in the 3Ps for Prevention Study to evaluate PrEP uptake and adherence. Nucleic acid amplification testing for Chlamydia trachomatis (CT) and Neisseria gonorrhoeae (NG) and a rapid test for Trichomonas vaginalis (TV) was conducted at screening and 6 months, and infections were treated. Symptom screening was performed at each visit. Predictors of incident STIs were analysed via Poisson regression to evaluate potential targeting of STI screening.

Results 200 AGYW were enrolled with a median age of 19 (IQR 17-21). At screening one-third of women tested positive for a curable STI: $25 \%$ CT, $11 \%$ NG, and 6\% TV, $98 \%$ of whom were asymptomatic. At month 6 , the incidence of a curable STI was 52/100 person-years (100py); CT 42/100py, NG 14/100py and TV 10/100py. Most incident STIs were diagnosed in women who did not have these infections at enrolment: $62 \%$ of 39 incident CT, $77 \%$ of 13 incident NG, and $89 \%$ of 9 incident TV infections. No significant associations were found between age, hormonal contraception or IPV and incident STIs.

Conclusion South African AGYW in a PrEP demonstration project had very high prevalence and incidence of asymptomatic curable STIs. Offering STI testing and treatment in PrEP programs is an important reproductive health service, and is valued by AGYW. Treatment of partners and innovative strategies to prevent STIs, including doxycycline post-exposure prophylaxis and vaccines, need to be evaluated in African AGYW.

Disclosure No significant relationships.

\section{P433 LOW HIV INCIDENCE AMONG WOMEN DIAGNOSED WITH SYPHILIS OR GONORRHEA DOES NOT SUPPORT PREP RECOMMENDATIONS, KING COUNTY, WA}

${ }^{1}$ Anna Berzkalns*, ${ }^{2}$ Lindley Barbee, ${ }^{2}$ Julia Dombrowski, ${ }^{2}$ Matthew Golden. ${ }^{1}$ Public Health Seattle and King County, HIV/STD Program, Seattle, USA; 'University of Washington, Medicine, Seattle, USA

\subsection{6/sextrans-2019-sti.519}

Background The November 2018 U.S. Preventive Services Task Force draft statement recommended clinicians offer HIV pre-exposure prophylaxis (PrEP) to women with recent syphilis (all stages) or gonorrhea diagnosis. We estimated HIV incidence among women in King County, WA following syphilis or gonorrhea diagnosis, and attempted to identify risk factors for HIV acquisition that might prompt clinicians to offer PrEP. Methods We matched public health HIV and sexually transmitted infection (STI) surveillance data for 2008-2018. Incidence rate was calculated using person-years (py) of follow-up, defined as time from initial STI diagnosis date to either HIV diagnosis date or end of study period. Women with prior or concurrent (within seven days of STI diagnosis) HIV diagnosis were excluded. Risk factors were identified from disease intervention specialists (DIS) interviews.

Results Between 2008-2018, 5,524 and 397 King County women were diagnosed with gonorrhea and syphilis, respectively. Over 1,705 py (median: 3.8) of follow-up, no incident HIV diagnoses occurred among women diagnosed with syphilis. Among women diagnosed with gonorrhea, 16 incident HIV diagnoses (incidence 0.06/100 py) occurred over 24,758 py (median: 3.7). Median time from gonorrhea to HIV diagnosis was 1.7 years (IQR: 0.5-3.0). DIS interviewed four (25\%) of the incident diagnoses following gonorrhea diagnosis; two (50\%) had risks identified: both reported injection 